\title{
Mesoscale energetics and flows induced by sea-land and mountain-valley contrasts
}

\author{
S. Federico ${ }^{1}$, G. A. Dalu ${ }^{2}$, C. Bellecci ${ }^{3}$, M. Colacino ${ }^{2}$ \\ ${ }^{1}$ CRATI S.c.r.l c/o University of Calabria, I-87036 Arcavata di Rende (CS) Italy, e-mail: federico@fermi.fis.unical.it \\ 2 IFA-CNR Tor Vergata, via Fosso del Cavaliere, n. 100, I-00133 Rome, Italy \\ ${ }^{3}$ S.T.F.E. Department, Engineering Faculty. University of Tor Vergata. Via di Tor Vergata, I-00133 Rome, Italy
}

Received: 19 March 1999 / Revised: 29 September 1999 / Accepted: 15 October 1999

\begin{abstract}
We study the relative importance of sea-land and mountain-valley thermal contrasts in determining the development of thermally forced mesoscale circulations (TFMCs) over a mountainous peninsula. We first analyse the energetics of the problem, and using this theory, we interprete the numerical simulations over Calabria, a mountainous peninsula in southern Italy. The CSU 3-D nonlinear numerical model is utilised to simulate the dynamics and the thermodynamics of the atmospheric fields over Calabria. Results show the importance of orography in determining the pattern of the flow and the local climate in a region as complex as Calabria. Analysis of the results shows that the energetics due to the sea-land interactions are more efficient when the peninsula is flat. The importance of the energy due to the sea-land decreases as the mountain height of the peninsula increases. The energy stored over the mountain gains in importance, untill it is released by the readjustment of the warm mountain air as it prevails over the energy released by the inland penetration of the sea breeze front. For instance, our results show that over a peninsula $100 \mathrm{~km}$ wide the energy over the mountain and the energy in the sea-land contrast are of the same order when the height of the mountain is about $700 \mathrm{~m}$, for a $1500 \mathrm{~m}$ convective boundary layer (CBL) depth. Over the Calabrian peninsula, the energy released by the hot air in the CBL of the mountain prevails over the energy released by the inland penetration of the sea air. Calabria is about $1500 \mathrm{~m}$ high and about $50 \mathrm{~km}$ wide, and the CBL is of the order of $1500 \mathrm{~m}$. The energy over the mountain is about four time larger than the energy contained in the sea-land contrast. Furthermore, the energetics increase with the patch width of the peninsula, and when its half width is much less than the Rossby radius, the MAPE of the sea breeze is negligible. When its half width is much larger than the Rossby radius, the breezes from the two opposing coastlines do not interact. Over Calabria peninsula, numerical simulations
\end{abstract}

Correspondence to: $\mathrm{S}$. Federico show that the flow is highly ageostrophic, and that the flow intensity increases from sunrise to reach its maximum in the afternoon but before sunset, which suggests that, in the late part of the day, the conversion of potential energy into kinetic energy is balanced by the dissipation.

Key words: Meteorology and atmospheric dynamics (climatology; mesoscale meteorology)

\section{Introduction}

In the early hours of the morning the sun warms the east-facing slopes first, then during the mid part of the day the south-facing slopes and the west-facing slopes in the afternoon. The local thermally driven winds follow the development of the convective boundary layer $(\mathrm{CBL})$, with some delay. Over a mountainous peninsula the convective boundary layer has different depths and altitudes; the resulting horizontal thermal gradients drive mesososcale flows. Over the slopes, the diabatic flux in the CBL drives a mountain-valley breeze, and, in addition, since a peninsula is bounded by the sea, the sea-land thermal contrast at the coast drives a sea-land breeze.

The scope of this study is to quantify the relative importance of the sea breeze and of the valley breeze over a peninsula. We evaluate analytically the mesoscale available potential energy (MAPE) due to the sea-land contrast, the mountain-valley contrast, and the mountain-sea contrast. We then use these results to examine the role of the sea-land and the mountain-valley breezes in the thermal convergence over Calabria. Calabria is a peninsula located in southern Italy, which ranges between $38^{\circ} 12^{\prime}$ and $40^{\circ}$, and between $16^{\circ} 30^{\prime}$ and $17^{\circ} 15^{\prime} \mathrm{E}$. On the west Calabria is bounded by the Tirrenian Sea, and on the south and on the east by the 
Ionian Sea. The topography (Fig. 1) is characterised by the ridges of the Apennines. From north to south, there are four main ridges: Catena Costiera, La Sila, Le Serre, and Aspromonte. There are four main peaks: Montalto in the Aspromonte, Pecoraro in the Serre, Botte Donato in the La Sila and Serra Dolce Dorme the north side of the Catena Costiera (coastal ridge). These mountains are steep with an altitude of $1500-2000 \mathrm{~m}$ or 1.5 to $2.0 \mathrm{~km}$ and a half width of few tens of kilometres. The pass of Marcellinara, a gap between La Sila and Le Serre, is located in the narrowest part of the Calabrian peninsula in the $\mathrm{W}-\mathrm{E}$ direction, its width is $30 \mathrm{~km}$ from coast to coast. In Calabria there are three main valleys close to the sea: Sibari to the east on the Ionian Sea, and Gioia Tauro and Lamezia to the west on the Tyrrhenian Sea.

Previous studies suggest that the local climate in Calabria is controlled by the sea breeze and by the upslope flow, which occur frequently under calm or nearly calm conditions and clear skies, typical of the warm season in Calabria (Baldi et al., 1997; Baldi et al., 1998). Local flows are strongly conditioned by the chief of the region, which plays a fundamental role in determining the location and the strength of the cumulus convection. Fair weather cloud distribution is strongly related to the specific morphology of the peninsula, which induces upslope flows and sea breezes, accompanied by vigorous convergence and upwelling over the crests of the ridges in the interior of the peninsula. Therefore it is interesting to examine the effects of sea-land and mountain-valley contrast because of their relevance in determining the local climate.

Three simulations are presented and compared with considerations on the energetics:

1. The sea-land breeze over a flat peninsula

2. The mountain-valley breeze

3 . The combination of the sea-breeze with the mountainvalley breeze

In the first case we consider no orography and a sealand contrast as if the Calabria peninsula were flat, in this case the forcing is only due to the sea-land contrast. In the second case the real topography of Calabria is introduced but we ignore the sea-land contrast (the sea is substituted by flat land), in this case the forcing is due to the mountain valley contrast. In the third case the real sea-land contrast and the real topography of Calabria are both present, in this case the forcing is due to the combined sea-land contrast and mountain-valley contrast.

\section{Energetics due to the sea-land contrast over a flat peninsula}

In order to evaluate the role of sea-land contrast in determining the local flow intensity, we evaluate the energetics over a flat peninsula. The mesoscale available potential energy, MAPE, is the energy difference

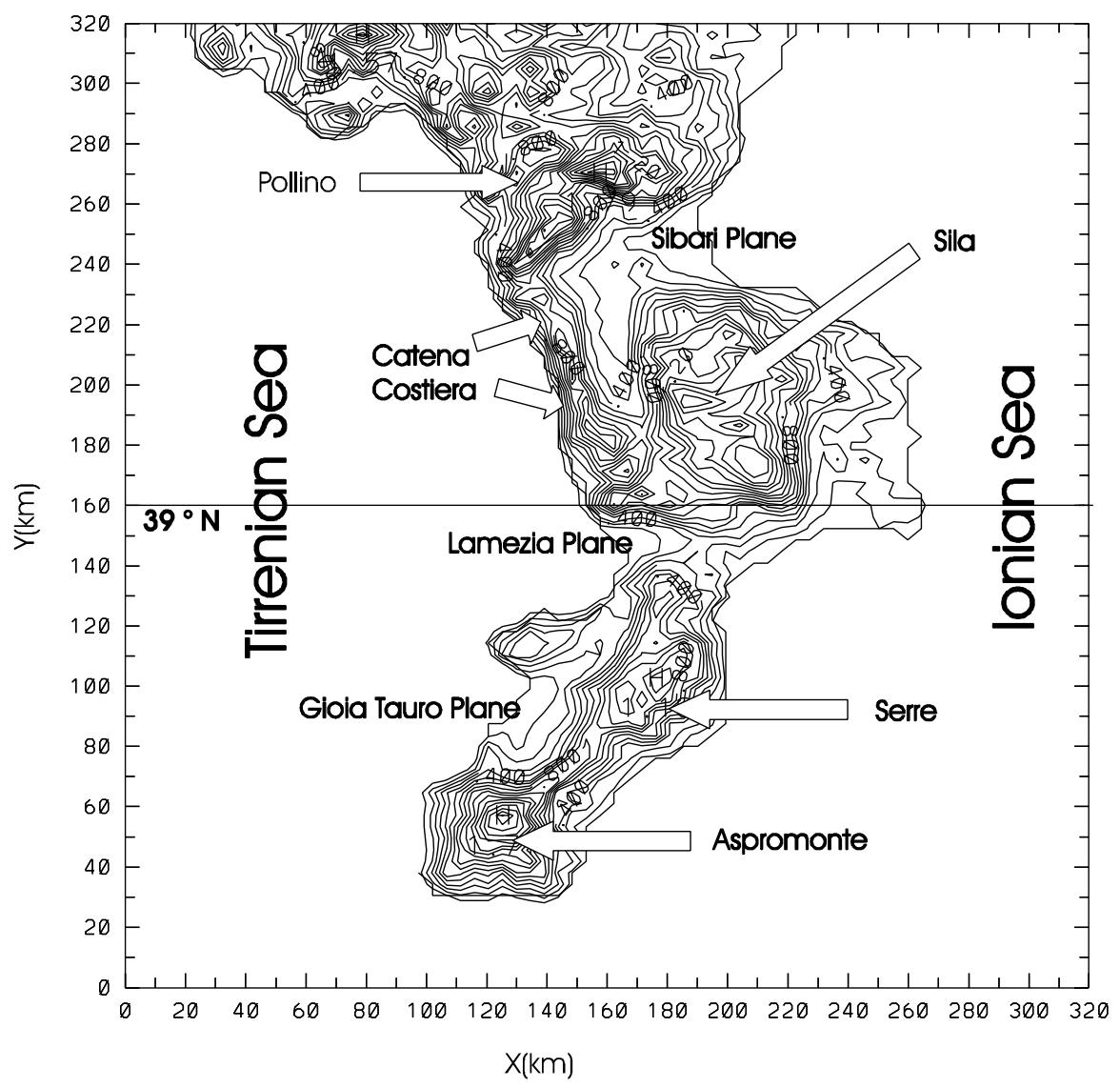

Fig. 1. Topography of Calabria 
between the initial and final configuration (Green and Dalu, 1980):

$$
\begin{aligned}
\text { MAPE } & =P E_{i}-P E_{f} \\
& =\int_{-(R+L)}^{R+L} \mathrm{~d} x \int_{0}^{h}\left(\frac{g \theta_{i}}{\Theta}-\frac{g \theta_{f}}{\Theta}\right) z \mathrm{~d} z .
\end{aligned}
$$

Here $2 L$ is the width of the peninsula, $h$ is the depth of the mesoscale flow, and $R$ is e-folding distance of the flow intensity from the coastline (Dalu and Pielke, 1989). $\theta_{i}$ is the initial diabatic temperature perturbation, and $\theta_{f}$ is the final temperature perturbation. In the final state the potential energy is lower, the difference is converted into kinetic energy of the mesoscale flow or dissipated. The sea-breeze stream-function, computed using linear theory is:

$$
\begin{aligned}
& \psi(x, z)=\psi_{0} H\left(\frac{z}{h_{0}}\right) \mathrm{e}^{-|x| / R_{0}} ; \\
& H\left(\frac{z}{h_{0}}\right)=\frac{1}{2 \pi}\left\{\frac{z}{h_{0}} \ln \left|\frac{z^{2}-h_{0}^{2}}{z^{2}}\right|+\ln \left|\frac{z+h_{0}}{z-h_{0}}\right|\right\} \\
& \psi_{0}=\frac{N_{0} h_{0}^{2}}{2} ; \quad R_{0}=h_{0} \frac{N_{0}}{f} .
\end{aligned}
$$

Here $N_{0}$ is the Brunt-Vaisala frequency and $f$ is the Coriolis parameter where $h_{0}$ is the depth of the CBL over the peninsula and $R_{0}$ is the Rossby radius. The seabreeze intensity has an e-folding distance from the coastline equal to a Rossby radius (Dalu and Pielke, 1993).

The stream-function over two adjacent lands with CBL of different depths, $h_{1}$ and $h_{2}$ respectively, is:

$\psi(x, z)=\frac{N_{0} h_{1}^{2}}{2} H\left(\frac{z}{h_{1}}\right) \mathrm{e}^{-|x| / R_{1}}-\frac{N_{0} h_{2}^{2}}{2} H\left(\frac{z}{h_{2}}\right) \mathrm{e}^{-|x| / R_{2}}$

Over a peninsula with the coastlines at $x=L$ and at $x=-L$, the stream-function is:

$$
\begin{aligned}
\psi_{\text {peninsula }}(x, z) & =\psi(x+L)+\psi(x-L) \\
& =\psi_{0} H\left(\frac{z}{h_{0}}\right)\left(\mathrm{e}^{-|x+L| / R_{0}}-\mathrm{e}^{-|x-L| / R_{0}}\right) ;
\end{aligned}
$$

The flow intensity, Eq. (4), plotted as a function of the width of the peninsula (Fig. 2) shows that, when $L<R_{0}$, the two sea-breeze cells in Eq. (3) interfere. Furthermore, when $L \ll R_{0}$, the breeze intensity becomes negligible, and, when $L \gg R_{0}$, the two breeze cells are independent, therefore we study the mesoscale energetics over a peninsula where $0<L<R_{0}$.

Then $R$ in Eq. (1), using Eqs. $(2,4)$, is given by:

$\psi_{\text {peninsula }}(x=-(R+L), z)=\psi\left(x=R_{0}, z\right)=\frac{\psi_{0}}{e} H\left(\frac{z}{h_{0}}\right)$

Figure $3 \mathrm{a}, \mathrm{b}$ shows the initial and final configuration of air masses for a flat peninsula. The MAPE is:

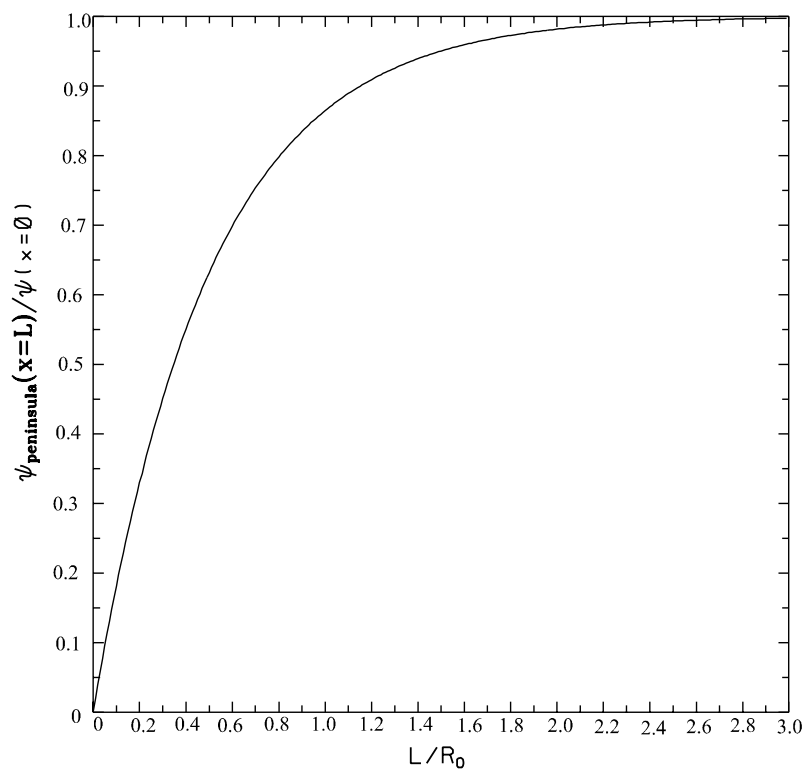

Fig. 2. Stream-function of two interacting breeze cells as a function of peninsula width. The stream-function of the peninsula is normalized to the stream-function of a single sea breeze cell

$$
\begin{aligned}
\text { MAPE }= & P E_{i}-P E_{f}=2 \int_{L}^{R+L} \mathrm{~d} x \int_{0}^{h_{i}} g \frac{\Theta_{z}}{\Theta}\left(h_{i}-z\right) z \mathrm{~d} z \\
& -2 \int_{0}^{R+L} \mathrm{~d} x \int_{0}^{h_{f}} g \frac{\Theta_{z}}{\Theta}\left(h_{f}-z\right) z \mathrm{~d} z \\
= & \frac{R N_{0}^{2} h_{i}^{3}}{3}-\frac{(R+L) N_{0}^{\prime 2} h_{f}^{3}}{3} .
\end{aligned}
$$

Here $N_{0}, N_{0}^{\prime}$ are the initial and the final Brunt-Vaisala frequencies, respectively, and $\Theta_{z}$ and $\Theta_{z}^{\prime}$ are the initial and final vertical potential temperature gradients, respectively. The final configuration and the final BruntVaisala frequency are determined by mass conservation and by thermodynamics:

$\left\{\begin{array}{l}N_{0}^{\prime 2}=N_{0}^{2} \frac{h_{i}}{h_{f}} \\ h_{f}=R \frac{h_{i}}{R+L}\end{array}\right.$

The mean sea-breeze intensity $\bar{U}$ is the square root of 2MAPE divided by the volume, $2(R+L) h_{i}$, involved in the adiabatic redistribution of air masses:

$\bar{U}=\left[\left(1-\frac{R}{R+L}\right) \frac{N_{0}^{2} h_{i}^{3}}{2(R+L)}\right]^{1 / 2}$

Figure $4 \mathrm{a}, \mathrm{b}$ shows the mesoscale available potential energy and the mean wind intensity as computed by Eqs. (7) and (8). The CBL heights are 1.0, 1.5 and $2.0 \mathrm{~km}$, respectively, $L$ ranges from 0 to $200 \mathrm{~km}$. The sea-breeze intensity grows as the boundary layer height increases and as the width of the peninsula increases, to reach its maximum when $L=R$, the flow intensity ranges from 2.5 to $5.5 \mathrm{~ms}^{-1}$ when the boundary layer ranges from 1 to $2 \mathrm{~km}$. 

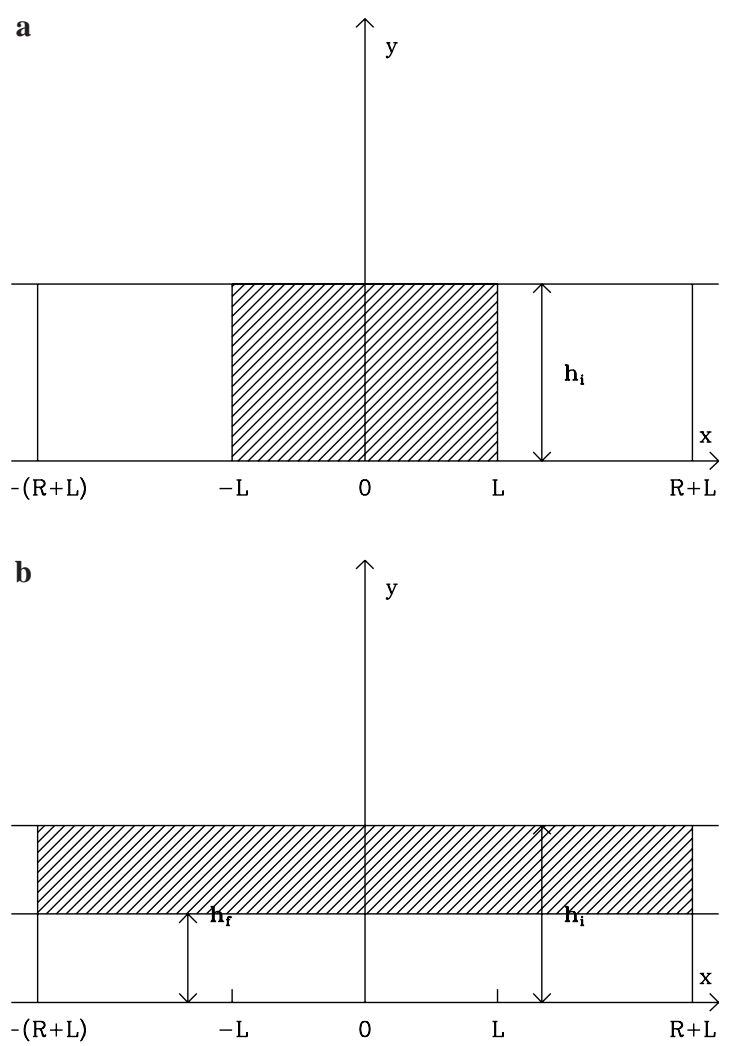

Fig. 3. a Air masses in a heated boundary layer over a flat peninsula. $h_{i}$ is the initial boundary layer depth, $2 L$ is the patch width and $R$ is the Rossby radius. $\mathbf{b}$ As in a but after the adiabatic redistribution of air masses. $h_{f}$ is defined by mass conservation. $R$ and $L$ as in a

Where $L \gg R$, the two sea-breezes are disconnected and independent. The sea-breeze MAPE in a semiinfinite land bounded by a semi-infinite sea is:

MAPE $=\frac{R N_{0}^{2} h_{i}^{3}}{3}$

\section{Energetics of the mountain-valley and the sea-mountain flow}

Figure $5 \mathrm{a}, \mathrm{b}$ shows the initial and final distributions of air masses over a land with a low mountain. Figure 5c, d shows the initial and final configurations over a land with a high mountain. When the land is bounded by the sea, i.e. the peninsula case, the CBL depth over the sea is zero. When the ridge is bounded by flat land, there is a $\mathrm{CBL}$ over the plain as well. In general the CBL depth over the mountain is different than the CBL over the plain.

\subsection{Energetics due to the sea-land contrast, where there is a mountain}

In this section, in the evaluation of the potential energy, we take as reference the temperature of the air initially
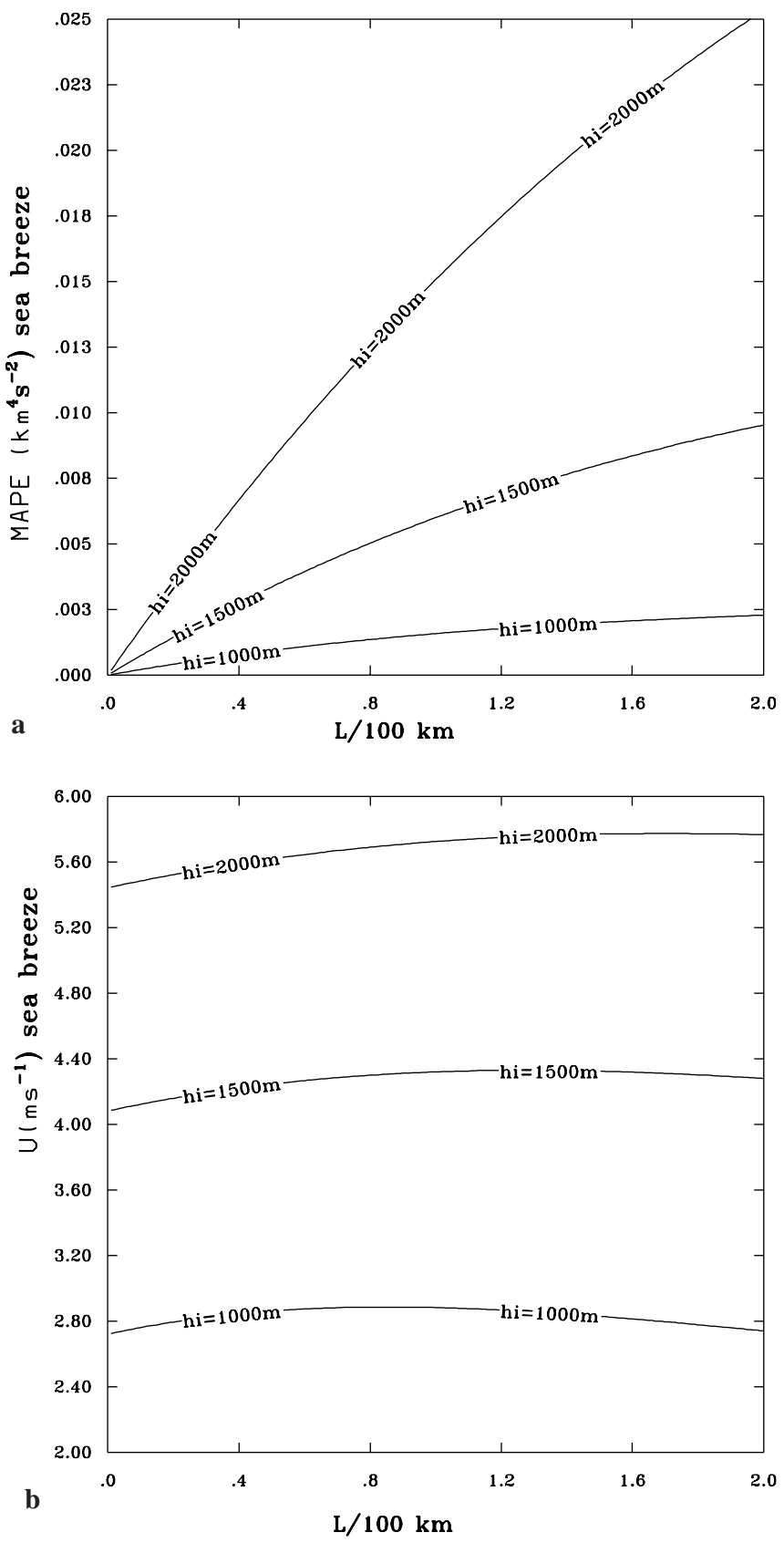

Fig. 4. a Mesoscale available potential energy for the sea-land contrast shown in Fig. 3a, b. The three curves refer to $h_{i}=1000 \mathrm{~m}$, $h_{i}=1500 \mathrm{~m}, h_{i}=2000 \mathrm{~m}$. $L$ as in Fig. 3a. b Mean wind velocity for the sea-land contrast shown in Fig. 3a, b. The three curves refer to $h_{i}=1000 \mathrm{~m}, h_{i}=1500 \mathrm{~m}, h_{i}=2000 \mathrm{~m} . L$ as in Fig. 3a

at $z=h_{1}$. The initial potential energy, due to the sealand contrast, $P E_{i-s e a}$, is:

$P E_{i-s e a}=2 \int_{L}^{R+L} \mathrm{~d} x \int_{0}^{h_{1}} g \frac{\delta \theta_{i}}{\Theta} z \mathrm{~d} z ; \quad \delta \vartheta_{i}=\Theta_{z}\left(h_{1}-z\right)$

where $h_{1}$ is the CBL depth and $\Theta_{z}$ is the initial potential temperature vertical gradient. The final potential energy, $P E_{f-s e a}$, is: 
$\mathbf{a}$

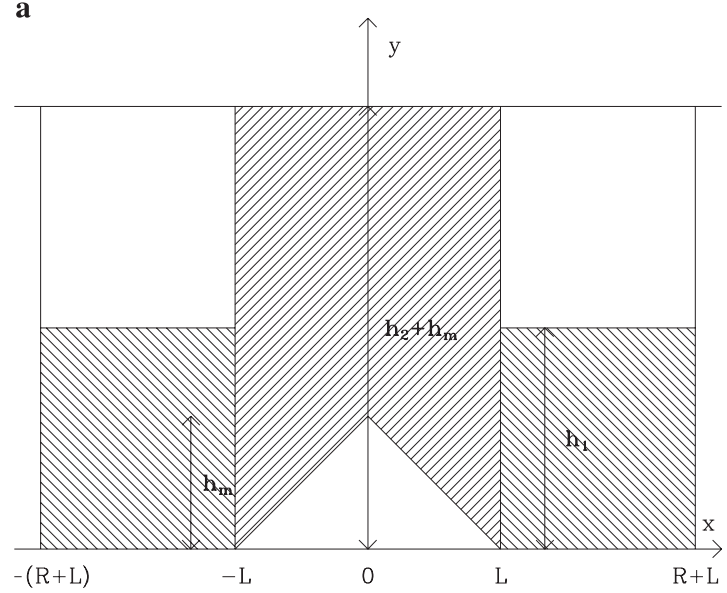

c

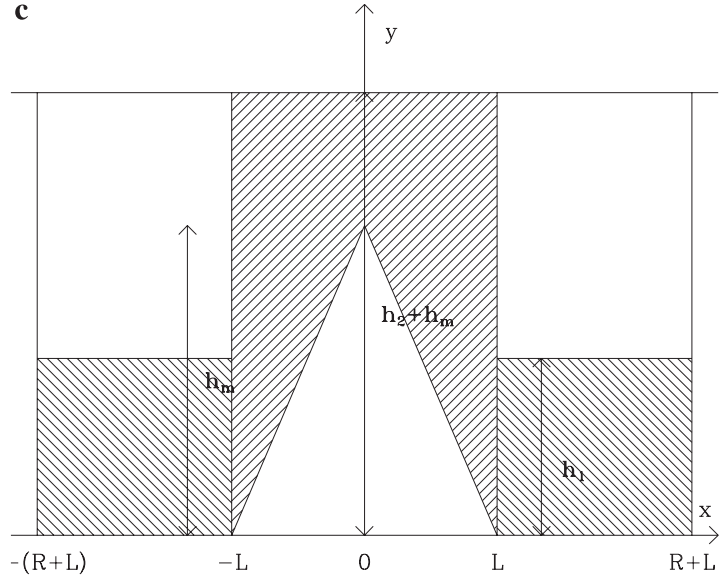

b

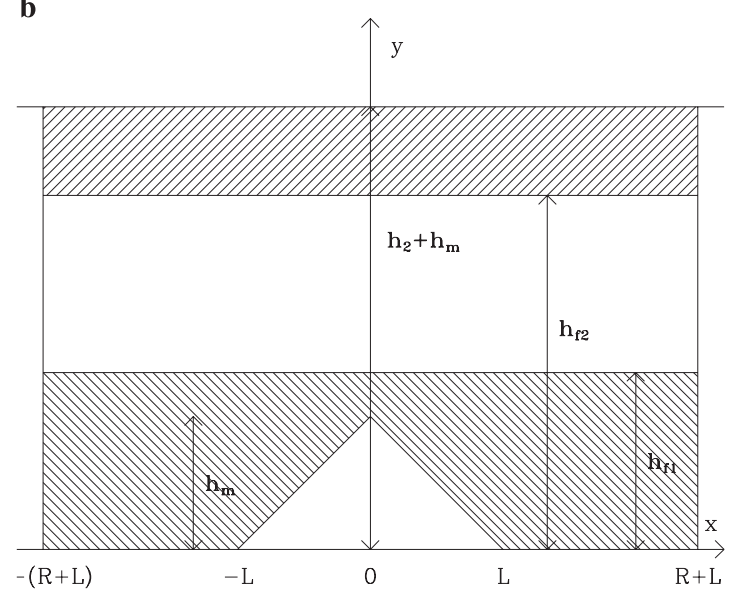

d

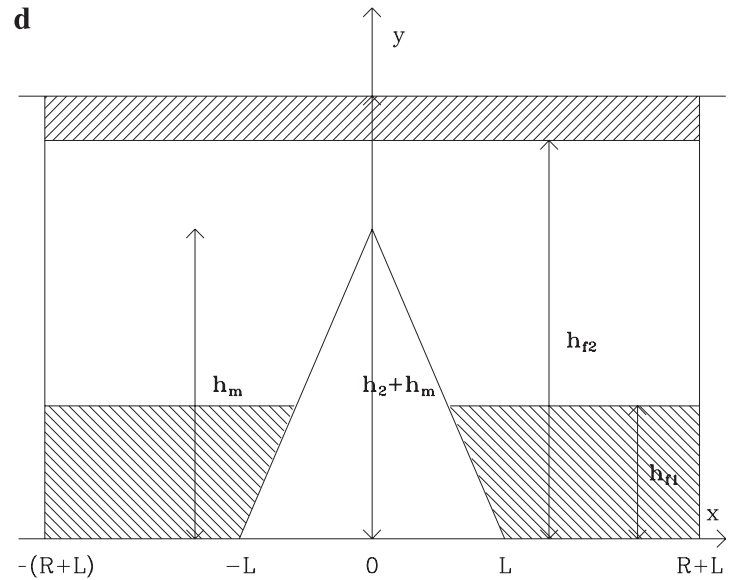

Fig. 5. a Air masses in two heated boundary layer over mountainous terrain, for a small mountain. $h_{m}$ is the mountain height. $R$ and $L$ as in Fig. 3a. $h_{2}$ is the boundary layer depth over the mountain and $h_{1}$ is the boundary layer depth over the flat terrain. b As in a but after an adiabatic redistribution so that $h_{m}<h_{f 1} . h_{f 1}$ and $h_{f 2}$ represent the final heights of the boundary layer $h_{1}$ and $h_{2}$, respectively. $R$ and $L$ as in Fig. 3a. c As in a but for a high mountain. d As in b but for $h_{m}>h_{f 1}$, for a high mountain
$P E_{f-\text { sea }}=2 \int_{0}^{R+L} \mathrm{~d} x \int_{h(x)}^{h_{f 1}} g \frac{\delta \theta_{f}}{\Theta} z \mathrm{~d} z ; \quad \delta \vartheta_{f}=\Theta_{z}^{\prime}\left(h_{f 1}-z\right)$

where $h_{f 1}$ is the final depth of the sea air, $\Theta_{z}^{\prime}=\frac{h_{1}}{h_{f 1}} \Theta_{z}$, and $h(x)$ is the mountain profile, and it is zero over the sea.

We first consider the energetics of a low mountain, $h_{m}<h_{f 1}$, and later we study the case of a high mountain, $h_{m}>h_{f 1}$. Referring to Fig. $5 \mathrm{a}$, b, i.e. to a low mountain, the initial potential energy, $P E_{i-s e a}$, is:

$P E_{i-\text { sea }}=2 \int_{L}^{R+L} \mathrm{~d} x \int_{0}^{h_{1}} g \frac{\Theta_{z}}{\Theta}\left(h_{1}-z\right) z \mathrm{~d} z=\frac{N_{0}^{2} h_{1}^{3} R}{3} ;$

$N_{0}^{2}=\frac{g \Theta_{z}}{\Theta}$

And the final potential energy $P E_{f-s e a}$ is:

$$
\begin{aligned}
P E_{f-\text { sea }}= & 2 \int_{0}^{R+L} \mathrm{~d} x \int_{0}^{h_{f 1}} g \frac{\Theta_{z}^{\prime}}{\Theta}\left(h_{f 1}-z\right) z \mathrm{~d} z \\
& -2 \int_{0}^{L} \mathrm{~d} x \int_{0}^{h_{m}\left(\frac{L-x}{L}\right)} g \frac{\Theta_{z}^{\prime}}{\Theta}\left(h_{f 1}-z\right) z \mathrm{~d} z \\
= & \frac{N_{0}^{\prime 2}}{3}\left[h_{f 1}^{3}(R+L)-h_{m}^{2} L\left(h_{f 1}-\frac{h_{m}}{2}\right)\right]
\end{aligned}
$$

The mesoscale available potential energy is MAPE $=$ $P E_{i-s e a}-P E_{f-\text { sea }}$. The final geometry is determined by mass conservation, while the final Brunt-Vaisala frequency is determined by the thermodynamics (Fig. 5b):

$\left\{\begin{array}{l}N_{0}^{\prime 2}=N_{0}^{2} \frac{h_{1}}{h_{f 1}} \\ h_{f 1}=h_{m} \frac{L}{2(R+L)}+R \frac{h_{1}}{R+L}\end{array}\right.$

In the case of a high mountain (Fig. 5c, d; $h_{f 1}<h_{m}$ ) the initial potential energy is given by Eq. (11) and the final $P E_{f-\text { sea }}$ is: 


$$
\begin{aligned}
P E_{f-\text { sea }}= & 2 \int_{L}^{R+L} \mathrm{~d} x \int_{0}^{h_{f 1}} g \frac{\Theta_{z}^{\prime}}{\Theta}\left(h_{f 1}-z\right) z \mathrm{~d} z \\
& +2 \int_{L\left(\frac{h_{m}-h_{f 1}}{h_{m}}\right)}^{L} \mathrm{~d} x \int_{0}^{h_{m}\left(\frac{L-x}{L}\right)} g \frac{\Theta_{z}^{\prime}}{\Theta}\left(h_{f 1}-z\right) z \mathrm{~d} z \\
= & \frac{N_{0}^{\prime 2}}{3}\left[h_{f 1}^{3} R+\frac{L h_{f 1}^{4}}{2 h_{m}}\right]
\end{aligned}
$$

Mass conservation and thermodynamics determine the final configuration and the Brunt-Vaisala frequency (Fig. 5d):

$$
\left\{\begin{array}{l}
N_{0}^{\prime 2}=N_{0}^{2} \frac{h_{1}}{h_{f 1}} \\
h_{f 1}=\frac{\left(R^{2} h_{m}^{2}+2 L h_{1} h_{m} R\right)^{\frac{1}{2}}-R h_{m}}{L}
\end{array}\right.
$$

\subsection{Energetics due to the CBL over the mountain}

In this section, in the evaluation of the potential energy, we take as reference the temperature of the air initially at $z=h_{2}+h_{m}$.

The initial potential energy, due to the hot air in the CBL over the mountain, is (Fig. 5a, c):

$$
\begin{aligned}
& P E_{i-\text { mountain }}=2 \int_{L}^{R+L} \mathrm{~d} x \int_{h_{1}}^{h_{1}+h_{m}} g \frac{\delta \theta_{i}}{\Theta} z \mathrm{~d} z ; \\
& \delta \vartheta_{i}=\Theta_{z}\left(h_{2}+h_{m}-z\right) .
\end{aligned}
$$

Here $h_{1}$ is the CBL depth, $\Theta_{z}$ is the temperature gradient, $h_{m}$ is the mountain height, and $h_{2}$ is the CBL depth over the mountain.

The initial potential energy, $P E_{i-\text { mountain }}$ Eq. (16), is:

$$
\begin{aligned}
P E_{i-\text { mountain }} & =2 \int_{L}^{R+L} \mathrm{~d} x \int_{h_{1}}^{h_{2}+h_{m}} g \frac{\Theta_{z}}{\Theta}\left(h_{2}+h_{m}-z\right) z \mathrm{~d} z \\
& =N_{0}^{2} R\left[\frac{\left(h_{2}+h_{m}\right)^{3}}{3}-\left(h_{2}+h_{m}\right) h_{1}^{2}+\frac{2}{3} h_{1}^{3}\right] .
\end{aligned}
$$

$P E_{i-\text { mountain }}$ is the same for a high or a small mountain.

For the evaluation of potential energy in the final configuration we first consider the energetics of a small mountain, $h_{m}<h_{f 1}$, and later the case of a high mountain, $h_{m}>h_{f 1}$.

For a small mountain the final potential energy $P E_{f-s e a}$ is:

$$
\begin{aligned}
P E_{f-\text { mountain }} & =2 \int_{0}^{R+L} \mathrm{~d} x \int_{h_{f 1}}^{h_{f 2}} g \frac{\Theta_{z}^{\prime \prime}}{\Theta}\left(h_{f 2}-z\right) z \mathrm{~d} z \\
& =N_{0}^{\prime \prime 2}(R+L)\left[\frac{h_{f 2}^{3}}{3}-h_{f 2} h_{f 1}^{2}+\frac{2}{3} h_{f 1}^{3}\right]
\end{aligned}
$$

The mesoscale available potential energy is MAPE $=P E_{i-\text { mountain }}-P E_{f-\text { mountain }}$. The final geometry is determined by mass conservation, while the final Brunt-Vaisala frequency is determined by the thermodynamics (Fig. 5b):

$$
\left\{\begin{array}{l}
N_{0}^{\prime \prime 2}=N_{0}^{2} \frac{h_{2}+h_{m}-h_{1}}{h_{f 2}-h_{f 1}} \\
h_{f 1}=h_{m} \frac{L}{2(R+L)}+R \frac{h_{1}}{R+L} \\
h_{f 2}=\frac{R\left(h_{2}+h_{m}\right)}{R+L}+\frac{L h_{m}}{2(R+L)}
\end{array}\right.
$$

For a high mountain (Fig. 5c, d; $h_{f 1}<h_{m}$ ) the initial potential energy is given by Eq. (17) and the final $P E_{f-\text { mountain }}$ is:

$$
\begin{aligned}
P E_{f-\text { mountain }}= & 2 \int_{0}^{R+L} \mathrm{~d} x \int_{h_{f 1}}^{h_{f 2}} g \frac{\Theta_{z}^{\prime \prime}}{\Theta}\left(h_{f 2}-z\right) z \mathrm{~d} z \\
& -2 \int_{0}^{L\left(\frac{h_{m}-h_{f 1}}{h_{m}}\right)} \mathrm{d} x \int_{h_{f 1}}^{h_{m}\left(\frac{L-x}{L}\right)} g \frac{\Theta_{z}^{\prime \prime}}{\Theta}\left(h_{f 2}-z\right) z \mathrm{~d} z \\
= & N_{0}^{\prime \prime 2}(R+L)\left[\frac{h_{f 2}^{3}}{3}-h_{f 2}^{2} h_{f 1}+\frac{2}{3} h_{f 1}^{3}\right] \\
& -N_{0}^{\prime \prime 2}\left[\frac{2 h_{f 1}^{3}\left(h_{m}-h_{f 1}\right)}{3 h_{m}}-\frac{h_{f 2} h_{f 1}^{2}\left(h_{m}-h_{f 1}\right)}{h_{m}}\right. \\
& \left.+\frac{h_{f 2}}{3}\left(\frac{h_{m}^{3}-h_{f 1}^{3}}{h_{m}}\right)-\frac{1}{6}\left(\frac{h_{m}^{4}-h_{f 1}^{4}}{h_{m}}\right)\right]
\end{aligned}
$$

Mass conservation and thermodynamics determine the final configuration (Fig. 5d) and the final BruntVaisala frequency:

$$
\left\{\begin{array}{l}
N_{0}^{\prime \prime 2}=N_{0}^{2} \frac{h_{2}+h_{m}-h_{1}}{h_{f 2}-h_{f 1}} \\
h_{f 1}=\frac{\left(R^{2} h_{m}^{2}+2 L h_{1} h_{m} R\right)^{\frac{1}{2}}-R h_{m}}{L} \\
h_{f 2}=\frac{R\left(h_{2}+h_{m}\right)}{R+L}+\frac{L h_{m}}{2(R+L)}
\end{array}\right.
$$

\subsection{Discussion on the energetics}

In the previous two sections we have shown the energetics of a sea-land and a mountain-valley contrasts where a mountain is located. Where there is a mountain bounded by a valley, i. e. a mountain-valley contrast, the total MAPE is given just by the second contribution (Sect. 3.2) because the isentropic air above the valley does not change its potential energy between the initial and final state. However, this air participates in the motion taking its kinetic energy from the potential energy of the warm air above the mountain. In the case of a mountainous peninsula, i. e. the sea-mountain contrast, the total MAPE is given by the sum of both contributions due to the redistribution of the air above the sea and the hot air above the mountain. 
In the following discussion the CBL depth over the sea is assumed to be zero. However when the ridge is bounded by a plain the depth of the CBL over the plain is assumed to be equal to the CBL depth over the mountain.

Figure $6 \mathrm{a}, \mathrm{c}$ and e shows the MAPE released by the sea breeze for a CBL depth of 1000, 1500, and $2000 \mathrm{~m}$. The different curves within the same frame refer to different heights of the mountain 0, 500, 1000, 1500, $2000 \mathrm{~m}$.

Figure 6a, c and e shows that the MAPE released by the sea breeze increases as the depth of the CBL increases. However, the sea breeze MAPE decreases as the height of the mountain increases, since the presence of a mountain acts as an obstacle to the inland penetration of the sea breeze. The barrier effect exerted by the mountain on the sea breeze increases as the mountain height increases.

Figure $6 \mathrm{~b}, \mathrm{~d}$ and $\mathrm{f}$ shows the MAPE released by the air which was initially in the CBL over the mountain. Different frames refer to different CBL depths 1000 , 1500 , and $2000 \mathrm{~m}$, different curves in the same frame refer to different heights of the mountain 500, 1000, $1500,2000 \mathrm{~m}$. The CBL depth over the ridge is assumed to be equal to the CBL depth over the flat land. Figure $6 \mathrm{~b}, \mathrm{~d}$ and $\mathrm{f}$ shows that the MAPE released by the warm air over the mountain increases as the depth of the CBL and as the height of the mountain increases. This MAPE is zero when the mountain height is zero. Therefore, there is for each width of a peninsula a height of the mountain at which the sea breeze MAPE equals the MAPE released by the mountain. With a mountain peninsula bounded by the sea, the total MAPE is the sum of the sea breeze MAPE with the MAPE released by the mountain air, as is shown in Fig. 7.

The MAPE released over a peninsula is computed by adding the sea breeze MAPE contribution to the MAPE contribution due to the hot air over the mountain, as is shown in Fig. $7 \mathrm{a}, \mathrm{c}$ and e. The different frames refer to different CBL depths 1000 , 1500 , and $2000 \mathrm{~m}$, while the different curves within the same frame refer to different heights of the mountain $0,500,1000,1500,2000 \mathrm{~m}$. The dashed curves refer to the flat peninsula case, Fig. 4a. In this case, the MAPE, released over a peninsula, increases as the boundary layer increases and as the peninsula width increases.

In the particular case of Calabria $\left(h_{1} \approx 1500 \mathrm{~m}\right.$ $L \approx 50 \mathrm{~km} h_{m} \approx 1500 \mathrm{~m}$ ) the energy released by the inland penetration of the sea is about a quarter of the energy released by the redistribution of the hot air over the mountain. The mean wind intensity is the square root of two times the kinetic energy density, $K E$. $K E$ is the ratio between the MAPE and the volume, $V$, of the air mass involved in the mesoscale flow:

$V=2(R+L)\left(h_{2}+h_{m}\right)-L h_{m}$

Figure $7 \mathrm{~b}, \mathrm{~d}$ and $\mathrm{f}$ shows the mean velocity field for the mountainous peninsula. The dashed curves show the case of a flat peninsula bounded by the sea, Fig. 4b.
The mean velocity increases as the boundary layer increases. In addition it is almost constant as a function of the peninsula width due to the increase of both the MAPE and the volume, $V$. In the specific case of Calabria $\left(h_{1} \approx 1500 \mathrm{~m}, L \approx 50 \mathrm{~km}, h_{m}=1500 \mathrm{~m}\right)$ the analytical computed mean wind is about $5.5 \mathrm{~ms}^{-1}$.

The development of the CBL over a flat terrain is:

$h(t)=\frac{2 Q_{0}}{N_{0}^{2}} \int_{0}^{t_{0}} q\left(t^{\prime}\right) \mathrm{d} t^{\prime}=h_{0}(1-\cos (\omega t))$

where $Q$ the diabatic bouyancy source:

$Q=Q_{0} \sin (\omega t) H e(h-z) r(x)=Q_{0} q(t) H e(h-z) r(x)$

The CBL over the mountain sides is a function of the slope and of its exposition. When the ridge runs northsouth, there is a time lag $\delta t$ between the west and the east side and a depth difference $\delta h$ :

$$
\begin{aligned}
\delta t=\frac{2 h_{m}}{\omega L} ; \quad \delta h=\frac{2 h_{m}}{L} h_{0} \sin (\omega t) ; \\
\omega=\frac{2 \pi}{\text { day }} ; \quad \sin \frac{h_{m}}{L} \approx \frac{h_{m}}{L}
\end{aligned}
$$

In Calabria, $\delta h$ is of the order of $100 \mathrm{~m}$. In addition, $\delta t$ is less than one hour and adds up to $T=\left(f^{2}+\lambda^{2}\right)^{-1 / 2}$, which is the delay of the mesoscale flow response to the CBL growth, $T$ is of the order of $1.5 \mathrm{~h}$ (Dalu et al., 1991). Therefore, the mesoscale flow is stronger on the east side in the morning hours, while in the afternoon it is stronger on the west side. When the ridge runs westeast, the CBL is deeper on the south side, the difference grows in time and reaches its maximum at sunset:

$\delta h=\frac{2 h_{m}}{L} h_{0}(1-\cos (\omega t))$

This effect becomes relevant when $\delta h$ is a sizeble fraction of $h_{1}$.

\section{The simulations}

Calabria is a narrow peninsula with high mountains, and, since its width is less than one Rossby radius in the east-west direction and two Rossby radii in the northsouth direction, we expect the induced atmospheric perturbation to be ageostrophic.

During the warm season, the local flow is dominated by the sea breeze and by the mountain valley breeze. Observations show that the sea and valley breezes merge in the early afternoon, producing a strong updrought. In order to examine the relative importance of the sea-land breeze and of the mountain-valley breeze over Calabria we simulate the dynamics and the thermodynamics using a 3-D nonlinear, hydrostatic, incompressible form of the Colorado State Mesoscale Model CSUMM. This model is described in Pielke (1974, 1984), Mahrer and Pielke (1977a, b) and McNider and Pielke (1981). The entire domain (Fig. 1) extends for $320 \mathrm{~km}$ in the N-S direction and $320 \mathrm{~km}$ in the E-W direction. The horizontal resolution is $\Delta x=\Delta y=3.33 \mathrm{~km}$ with 16 unevenly spaced vertical levels, which extend from ground to 

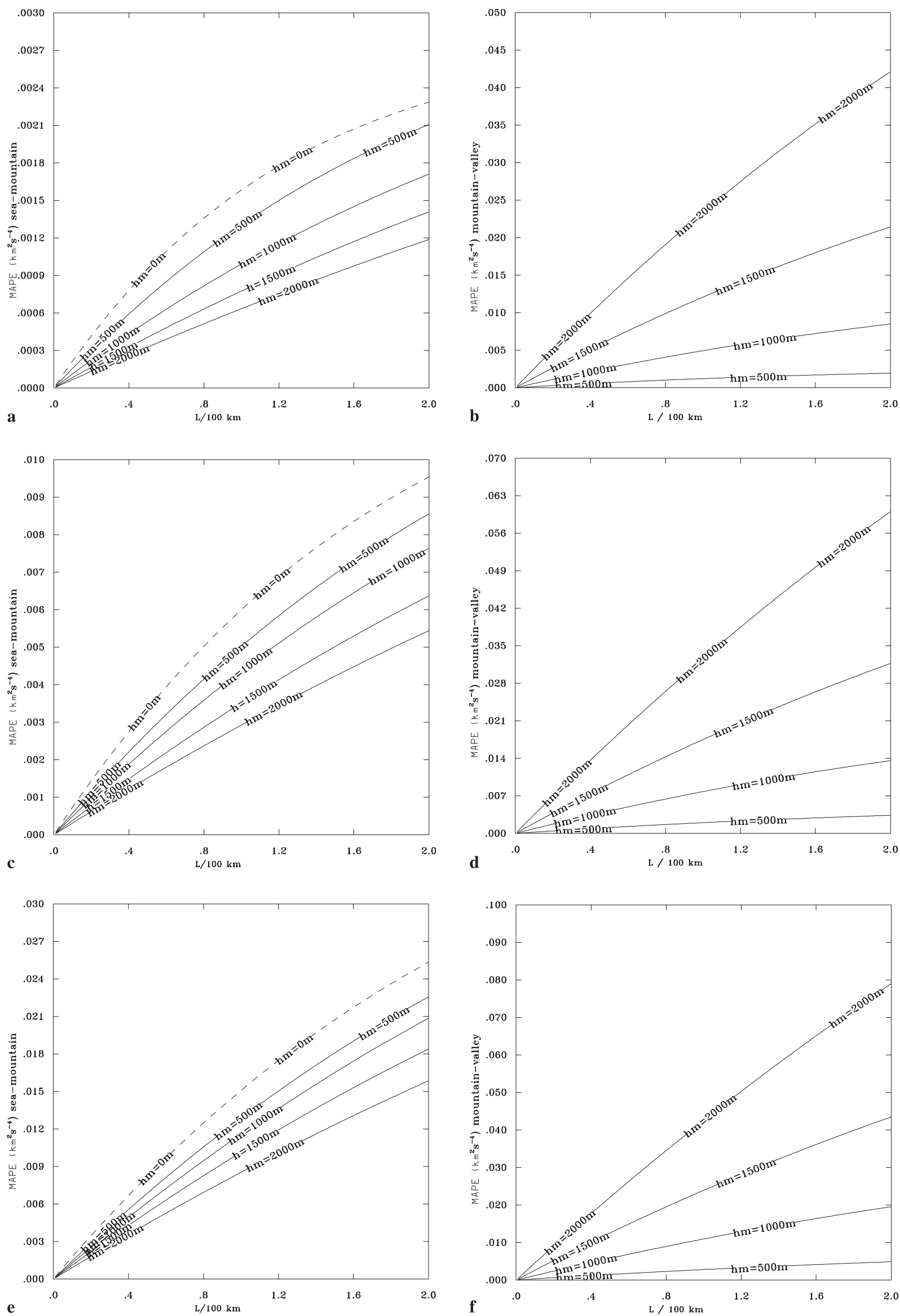
Fig. 6a-f. Mesoscale available potential energy for sea-land and mountain-valley contrasts in the mountainous terrain shown in Fig. 5a-d. a, $\mathbf{c}$ and e refer to the sea-land contrast and correspond to different heights of the CBL $h_{1}=1000, h_{1}=1500, h_{1}=2000 \mathrm{~m}$. b, d and $\mathbf{f}$ brefer to the mountain-valley contrast and correspond to different heights of the CBLs $h_{1}=h_{2}=1000, h_{1}=h_{2}=1500, h_{1}=$ $h_{2}=2000 \mathrm{~m}$. Different curves in the same frame represent different mountain heights, $h_{m}=500, h_{m}=1000, h_{m}=1500, h_{m}=2000 \mathrm{~m}$. Dashed lines refer to the flat terrain case, Fig. $4 \mathrm{a}$, and are shown for completeness

$13000 \mathrm{~m}$, with a dissipative layer above $7000 \mathrm{~m}$. The time step is $15 \mathrm{~s}$ and the integration time is $24 \mathrm{~h}$, starting from sunrise. A pre-run enables balancing of the meteorological fields with the lower boundary drag due to the topography and to the surface roughness. An initial weak environmental flow is assumed to jumpstart the surface fluxes, which allows us to initialise the friction velocity $u^{*}, \vartheta^{*}$, and $q^{*}$.

As reported in previous studies (Baldi et al., 1997), and, the (Baldi et al., 1997, 1998) climatic conditions for diabatic driven local flows are more favourable in summer, therefore in the next sections we report results for the 16, July, under calm large-scale winds and clear sky conditions, as often observed in Calabria in summer and sometime' in winter as well (Colacino, 1992), and (Colacino et al., 1997). We simulate the atmospheric flow in three different configurations.

1. We consider a flat peninsula and a sea-land contrast as in a Calabria with no topography.

2. We introduce the real topography of the Calabria peninsula but neglect the sea-land contrast, the sea is substituted by flat land.

3 . The real land-sea contrast and the real topography of Calabria are both present.

\subsection{Sea breezes simulations}

In this section we discuss the results for a run in which the sea-land contrast is introduced as if the Calabria peninsula had a uniform topography of $10 \mathrm{~m}$ a.s.l. (flat terrain).

We show results when the large-scale flow is weak $u_{g}=0.0 \mathrm{~ms}^{-1}, v_{g}=0.5 \mathrm{~ms}^{-1}$, the soil is rather dry and the static atmospheric stability is weak, $\beta=2 \mathrm{Kkm}^{-1}$.

The extent of Calabria in the W-E direction is less than one Rossby radius, therefore the Ionian and Tirrenian Sea breeze fronts meet, in the early afternoon, in the centre of the region. They define two climate axes in the north and south part of the region.

Figure 8 shows the wind vector at 16:00 LST at $75 \mathrm{~m}$ above the ground. A strong convergence of air masses in the centre of the region is evident. The maximum wind intensity is about $5 \mathrm{~ms}^{-1}$, roughly half of the maximum wind intensity of the case in which the real orography of Calabria is present.

This result is in good agreement with our considerations on the energetics, which show the important contribution of a mountain in determining the strength of local flows. Indeed the difference between the simulated mean field intensity and the one shown in Fig. 10 (i.e. sea-mountain contrast) is remarkably high. The mean flow simulated is less intense than that computed using the energetics. This difference is due to the fact that in the numerical model both physical and numerical dissipations are present.

\subsection{Simulations of the mountain-valley and of the sea-mountain flows}

First we discuss the results concerning a run in which the real topography of the Calabria peninsula is introduced, and the sea-land contrast is not considered. Then we present the results of a run in which the real topography and the real sea-land contrast of the Calabria peninsula are both introduced. The meteorological and soil parameters are as in the previous case. Figure 9 shows the wind vector at 16:00 LST and at $75 \mathrm{~m}$ above the ground in the case of mountain-valley contrast. The simulated maximum wind intensity is about $7 \mathrm{~ms}^{-1}$, showing less intense kinetic energy when locally compared to the case in which both forcings are present. These results are in agreement with our energetics considerations and show that the orographic forcing is, in the case of the Calabria peninsula, larger than the forcing due to sea-land contrast in determining local mesoscale flows.

In the case of sea-mountain contrast (Fig. 10), i.e. both forcings are considered, there is a strong convergence in the centre of the region. The simulated flow intensity maximum is larger than $9 \mathrm{~ms}^{-1}$ and there are four main convergence peaks, associated with the four main mountains of the peninsula. There is also evidence of two climate axes associated in the north and south part of the peninsula with the ranges Catena Costiera and Appennino Calabrese, respectively.

\section{Conclusions}

The present study investigates the relative role of the sea-land contrast and of the mountain-valley contrast in determining the local thermal convergence pattern over a mountainous peninsula with particular reference to Calabria. We use an analytical and numerical approach to study the problem. Our results show that the topography plays a fundamental role in determining the mesoscale flows. Indeed the presence of mountains highly enhances the wind intensity. In particular, for the Calabria peninsula, the importance of the mountainvalley contrast is larger than the sea-land contrast, therefore the local climate is due to the presence of the sea but also to its high topography. Our energetic analysis is in good agreement with the order of magnitude of the simulated fields. The simulated mean wind is less intense than that predicted using MAPE. This is related to the particular morphology of Calabria peninsula, which is very rugged and less than one Rossby radius wide. Therefore, energy losses due to 

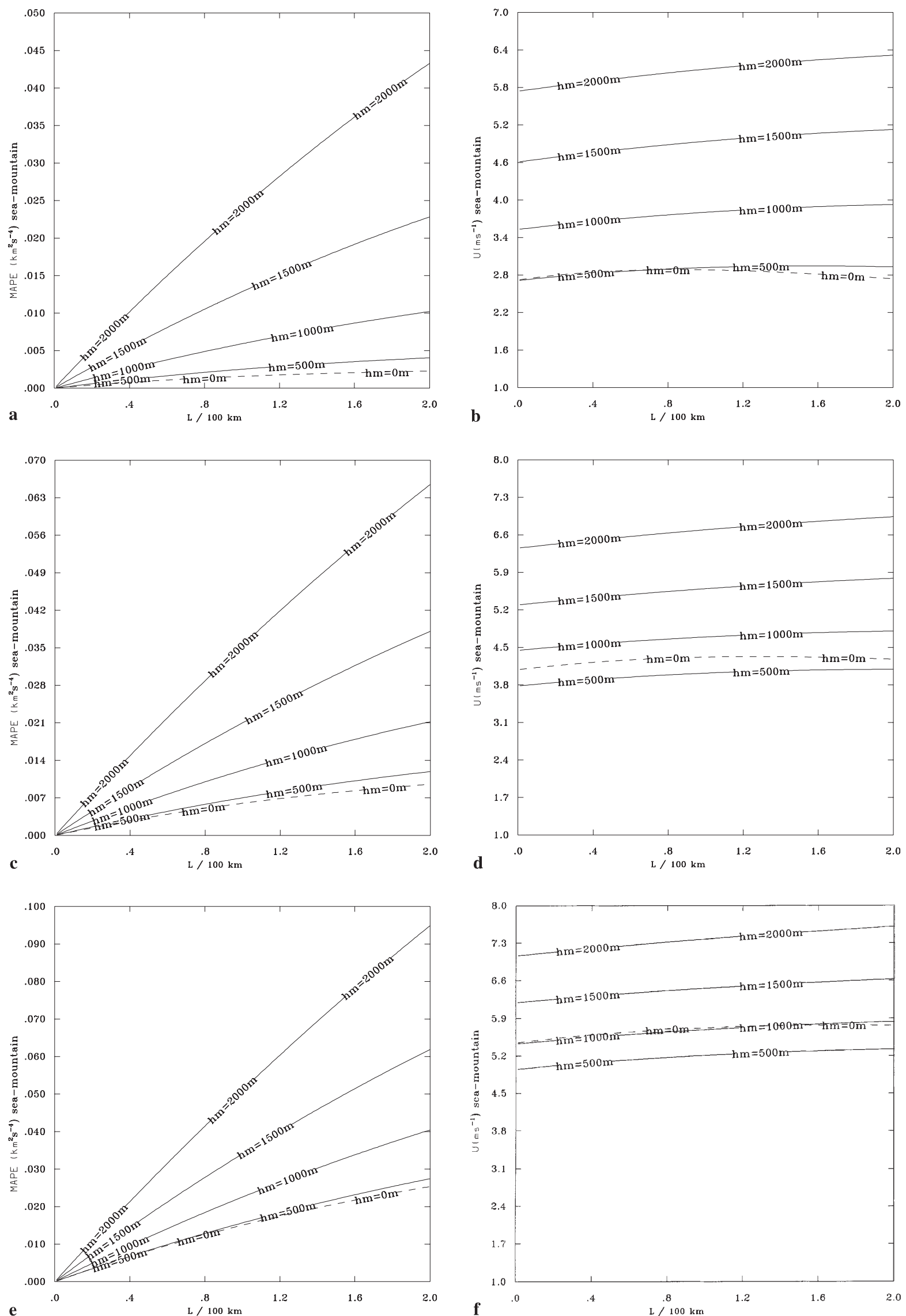


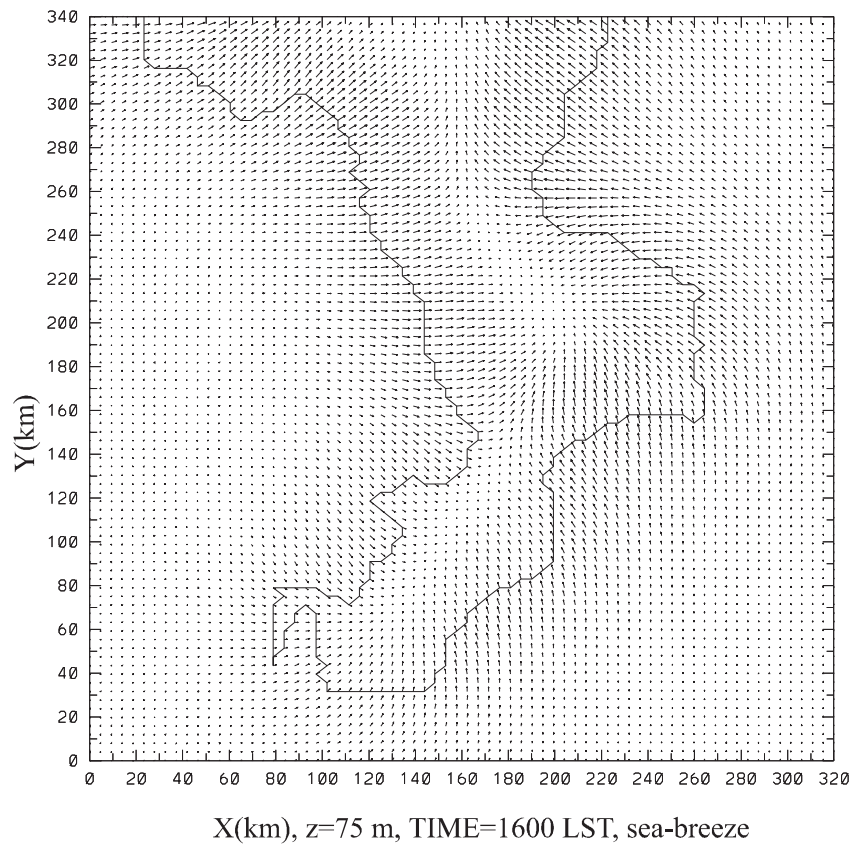

Fig. 8. Wind vector at $75 \mathrm{~m}$ above the ground simulated on July 16 at 16:00 LST for the sea-breeze case. $u_{G}=0.0 \frac{\mathrm{m}}{\mathrm{s}}, v_{G}=0.5 \frac{\mathrm{m}}{\mathrm{s}}, \beta=\frac{2 \mathrm{~K}}{\mathrm{~km}}$

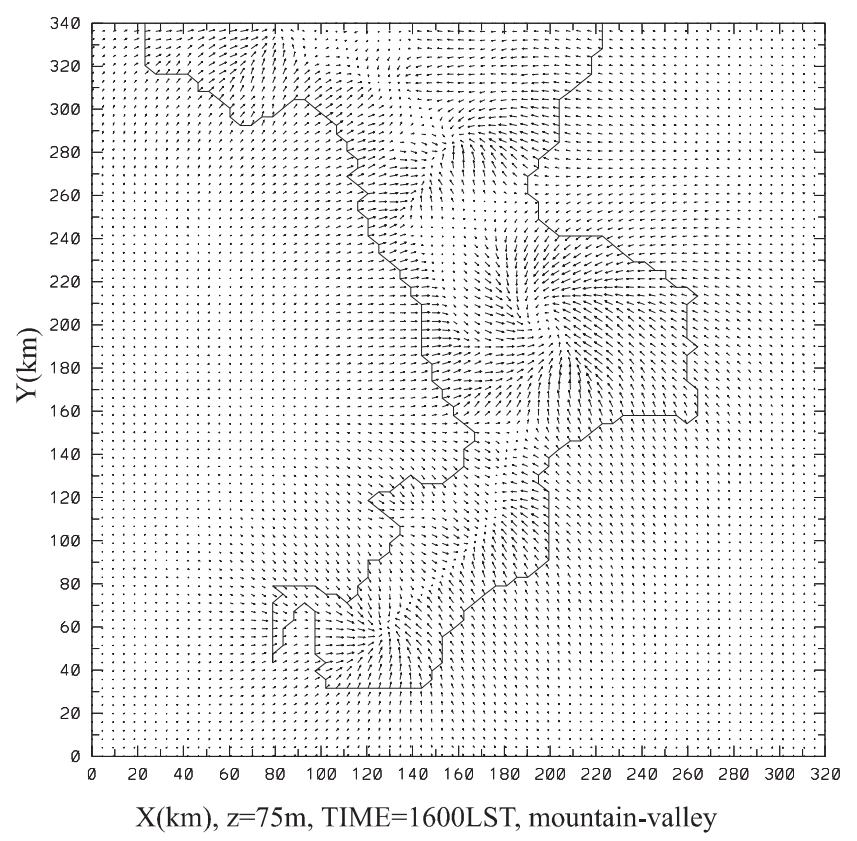

Fig. 9. As in Fig. 8 but for the mountain-valley flow

Fig. 7a-f. Mesoscale available potential energy and mean wind when both contrasts, i.e. sea-land and mountain-valley, are present (Fig. 5a-d). a, c and e refer to the MAPE and correspond to CBL heights of $h_{2}=1000, h_{2}=1500, h_{2}=2000 \mathrm{~m}$. b, d and $\mathbf{f}$ refer to the mean velocities for $h_{2}=1000, h_{2}=1500, h_{2}=2000 \mathrm{~m}$. Different curves in the same frame represent different mountain heights $h_{m}=500, h_{m}=1000, h_{m}=1500, h_{m}=2000 \mathrm{~m}$. Dashed lines refer to the flat terrain case, Fig. $4 a$, and are shown for completeness

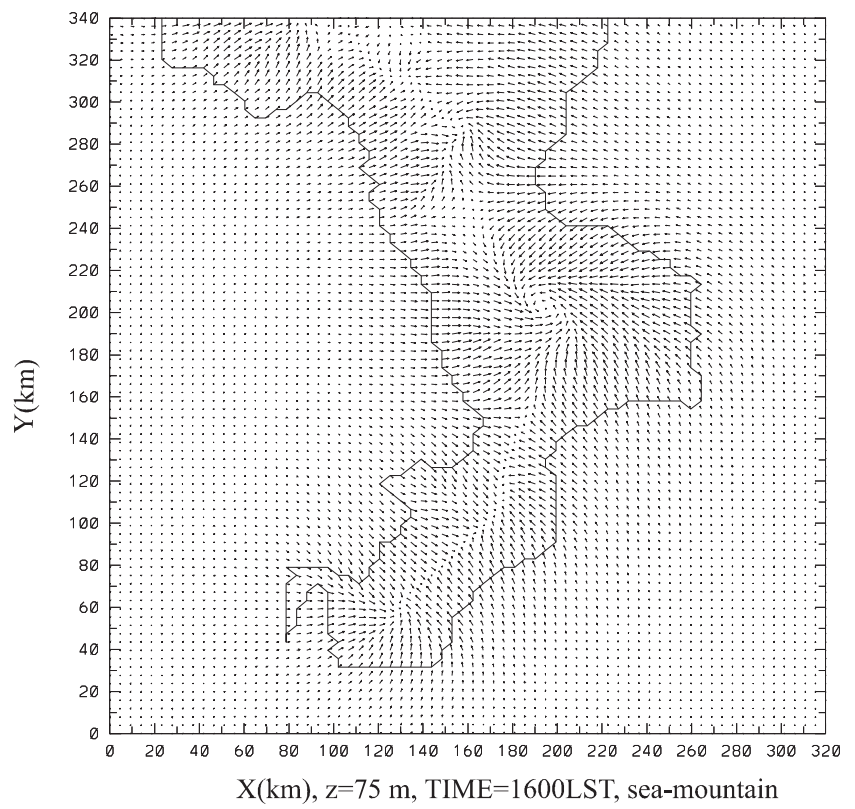

Fig. 10. As in Fig. 8 but for the sea-mountain flow

friction and dissipation are important, furthermore, since the circulation is highly ageostrophic, the breezes do not reach maturity and not all the potential energy is released. In fact, our MAPE evaluation of the mean wind is made considering an inviscid fluid, capable of releasing all the available potential energy. However, the relative contributions, i.e. the sea breeze, mountainvalley breeze and the combination of the sea breeze with the mountain-valley breeze in the simulated case and in the theoretical case are well reproduced. Despite the limitations of the numerical model in which there is dissipation due to the physics and also dissipation due to the numerical scheme, and considering also the limitations of the energetics theory which is non dissipative and highly efficient in releasing the potential energy, both approaches are useful in gaining knowledge of mesoscale flows.

Acknowledgements. S. Federico acknowledges the support of INEA, project POM-MISURA2, contract A05. G.A. Dalu acknowledges the support of ASI. We are in debt to the reviewers for their suggestions and for their contributions in improving this work. We are grateful to P. Aversa, and J. D. Dalu in their help for writing the paper and draughting the figures.

Topical Editor D. J. Webb thanks B. W. Atkinson and another referee for their help in evaluating this paper.

\section{References}

Baldi, M., M. Colacino, G. A. Dalu, E. Piervitali, and Z. Ye, Simulazioni a mesoscala su regioni a topografia complessa: applicazioni alla Calabria, IFA CNR, Roma R.I. 97-1, 1997.

Baldi, M., M. Colacino, G. A. Dalu, E. Piervitali, and Z. Ye, Thermally forced atmospheric flow over complex terrain in Southern Italy, Il Nuovo Cimento, 21C (4), 417-437, 1998.

Colacino, M., Mediterranean meteorology, In winds and currents of the Mediterranean basin, Proc. NATO-ASI, Ed H. Charnock. Reports in Meteorology and Oceanography 40, 1-38. Harvard University Press, USA, 1992. 
Colacino, M., M. Conte, and E. Piervitali, Elementi di climatologia della Calabria, IFA CNR Roma, 1997.

Dalu, G. A., and R. A. Pielke, An analytical study of the sea breeze, J. Atmos. Sci., 46, 1815-1825, 1989.

Dalu, G. A., and R. A. Pielke, Vertical fluxes generated by mesoscale atmospheric flow induced by thermal inhomogeneities in the PBL, J. Atmos. Sci., 50, 919-926, 1993.

Dalu, G. A., R. A. Pielke, R. Avissar, G. Kallos, M. Baldi, and A. Guerrini, Linear impact of thermal inhomogeneities on mesoscale atmospheric flow with zero synoptic wind, Ann. Geophys., 9, 641-647, 1991.

Green, J. A. S., and G. A. Dalu, Mesoscale energy generated in the boundary layer, Q. J. R. Meteorol. Soc., 106, 721-728, 1980 .
Mahrer, Y., and R. A. Pielke, A numerical study of the air flow over irregular terrain, Contr. Atmos. Phys., 50, 98-113, 1977a.

Mahrer, Y., and R. A. Pielke, The effects of topography on sea and land breezes in a two-dimensional numerical model, Mon. Weather Rev., 105, 1151-1162, 1977b.

McNider, T. R., and R. A. Pielke, Diurnal boundary-layer development over sloping terrain, J. Atmos. Sci., 38, 2198-2212, 1981.

Pielke, R. A., A three-dimensional numerical model of the sea breezes over South Florida, Mon. Weather Rev., 102, 115-139, 1974.

Pielke, R. A., Mesoscale meteorogical modeling, Academic Press, New York, 1984

Pielke, R. A., and C. L. Martin, The derivation of a terrainfollowing coordinate system for use in a hydrostatic model, J. Atmos. Sci., 38, 1708-1713, 1981. 